\title{
Impact of updated recommendations on acetylsalicylic acid use for primary prevention of cardiovascular disease in Canada: a population-based survey
}

\author{
Myriam Khalili MD, Fanny Lepeytre MD, Jason Robert Guertin PhD, Rémi Goupil MD, \\ Stéphan Troyanov MD, Josée Bouchard MD, François Madore MD
}

\section{Abstract}

Background: The debate over acetylsalicylic acid (ASA) therapy for primary prevention of cardiovascular disease (CVD) has recently resurfaced, but scarce data are available on prophylactic ASA use in Canada for this purpose. This study aimed to evaluate the prevalence and factors associated with ASA use, and the potential impact of implementing the most recent (2016) US Preventive Services Task Force recommendations for primary CVD prevention in a Canadian setting.

Methods: We performed a cross-sectional analysis using data from the CARTaGENE study, which included a representative sample ( $n=20004)$ of the 2018 general population of the province of Quebec. We assessed eligibility for ASA treatment using US Preventive Services Task Force criteria (age 50-69 yr, no past history of myocardial infarction or stroke, and 10-year risk of CVD of at least $10 \%$ ). We extrapolated to the entire 2018 Quebec population the number of people who would need to start ASA treatment.

Results: A total of 6231 respondents in the CARTaGENE study (54.2\% of those aged 50-69 yr with no prior history of CVD) were found to be potentially eligible for ASA use for primary CVD prevention. Of the $6231,1379(22.1 \%)$ were receiving prophylactic ASA treatment. Factors found to be related to ASA use included age, male sex, regular medical visits, lower education level, obesity, hypertension, diabetes and dyslipidemia. Income and smoking status were not found to be significantly associated with ASA use. Our results indicate that 885261 people would potentially have started ASA treatment if the US Preventive Services Task Force recommendations had been implemented in Quebec in 2018.

Interpretation: Prevalent ASA use for primary CVD prevention was low. Implementation of the 2016 US Preventive Services Task Force recommendations would require initiating ASA treatment in a substantial proportion of people, with undetermined potential benefits.

\begin{abstract}
$\longrightarrow$ ardiovascular disease (CVD) is the leading cause of mortality worldwide, ${ }^{1}$ accounting for $31 \%$ of all deaths in 2016. In Canada, CVD was responsible for 66922 deaths in 2017..$^{2}$ Improving cardiovascular health is a major target for research and prevention programs, and antiplatelet therapy has become one of the main strategies to reduce the risk of CVD. The benefits of low-dose acetylsalicylic acid (ASA) in secondary CVD prevention are well established. ${ }^{3,4}$ In primary prevention, however, there is no clear consensus on ASA use, as the evidence regarding its benefits remains uncertain. The debate over ASA therapy for primary CVD prevention has recently resurfaced, fuelled by trials such as ASCEND (A Study of Cardiovascular Events iN Diabetes) ${ }^{5}$ and ARRIVE (A Study to Assess the Efficacy and Safety of Enteric-Coated Acetylsalicylic Acid in Patients at Moderate Risk of Cardiovascular Disease), ${ }^{6}$ which showed minimal cardiovascular benefits from its use.

The American College of Cardiology and the American Heart Association recently issued a joint guideline on primary prevention of CVD in which they state that low-dose ASA might be considered for adults aged 40-70 years at higher
\end{abstract}

risk for CVD who do not appear to be at increased risk for bleeding. ${ }^{7}$ Risk factors for bleeding that need to be assessed for include a history of previous gastrointestinal bleeding or peptic ulcer disease, bleeding at other sites, thrombocytopenia, coagulopathy, chronic kidney disease and concurrent use of other medications that increase bleeding risk, such as nonsteroidal anti-inflammatory drugs, steroids, direct oral anticoagulants and warfarin. Likewise, the most recent (2016) US Preventive Services Task Force recommendations suggest initiating ASA treatment in adults aged 50-59 years with a $10 \%$ or greater 10 -year CVD risk given that the benefits probably outweigh the risks in these people. ${ }^{8}$ For adults aged 60-69 years with increased CVD risk, the decision should be

\section{Competing interests: None declared.}

This article has been peer reviewed.

Correspondence to: François Madore, f.madore@umontreal.ca

CMAJ Open 2020. DOI:10.9778/cmajo.20190105 
individualized, but the benefits probably slightly outweigh the risks according to these recommendations. ${ }^{8}$

Investigators in the United States and other countries have studied the prevalence of ASA use for primary prevention, ${ }^{9-12}$ but similar data are scarce in Canada. In the present analysis, we first evaluated the frequency of ASA use for primary CVD prevention and factors associated with its use in a large population-based survey. We then sought to evaluate the potential impact of implementing the 2016 US Preventive Services Task Force recommendations by extrapolating to the entire general population the number of people who would need to start ASA treatment.

\section{Methods}

\section{Design and study population}

We performed a cross-sectional study using data from 20004 randomly selected people aged 40-69 years from the Quebec general population included in the CARTaGENE cohort. ${ }^{13}$ Given the growing burden of chronic disorders, the CARTaGENE cohort was set up as a large biobank and databank so that determinants of diseases, including environmental, lifestyle and genetic factors, could be uncovered. This was a complex sample survey, and, overall, the final cohort was representative of the general population when compared with characteristics obtained from the 2006 Canadian census. Comparison of sociodemographic characteristics between the CARTaGENE cohort and the general population showed good concordance except that CARTaGENE participants were slightly more educated and ethnic minorities were slightly overrepresented. ${ }^{13}$

\section{Data sources}

Participants were recruited from August 2009 through October 2010 by means of a provincial government health insurance database. Participants completed an extensive interviewer-administered questionnaire inquiring about health, lifestyle and sociodemographic characteristics at one of the assessment sites. Anthropometric measurements, blood pressure measurements and blood sampling were performed at the time of the interview. Additional details on questionnaire domains, with sources of data, physical measurements and biochemical measures that were used for the study, are provided in Appendix 1 (available at www.cmajopen.ca/content/ 8/1/E41/suppl/DC1). A detailed description of CARTaGENE methods has been published elsewhere. ${ }^{13}$

\section{Eligibility for acetylsalicylic acid therapy}

All participants were evaluated for eligibility for ASA therapy for primary CVD prevention. Cardiovascular disease was defined as a self-reported history of myocardial infarction, angina or stroke. For all participants without a history of CVD, 10-year CVD risk was estimated with the Framingham Risk Score,${ }^{14}$ taking into account the participant's age, sex, total and high-density lipoprotein cholesterol values, systolic blood pressure, use of antihypertensive drugs, presence of diabetes and smoking status. Based on the 2016 US Preventive
Services Task Force recommendations,${ }^{8}$ participants aged 50-69 years with no past history of myocardial infarction or stroke and with a 10-year CVD risk of at least $10 \%$ were considered eligible for daily ASA use. Participants for whom a Framingham Risk Score could not be calculated because of missing data were excluded from the analyses, but multiple imputation for missing values was performed to evaluate the impact on study results. We also analyzed the number of participants taking anticoagulant drugs to estimate the number of candidates eligible for ASA treatment who might have a contraindication to its use. We did not exclude these participants from the analyses. Finally, we estimated the number of people who would need to start ASA treatment by extrapolation to the Quebec population using 2018 population estimates. ${ }^{15}$

\section{Measures}

Information was collected for all participants on the presence of a self-reported history of CVD, hypertension, diabetes or dyslipidemia, and for corresponding treatments (i.e., use of antihypertensive drug $[\mathrm{s}]$, antidiabetic agent $[\mathrm{s}]$ or statin[s]). Participants were asked to give the name of all prescribed and nonprescribed drugs they were using at the time of the interview and to provide, if possible, the drug containers so the information could be validated. For analyses, participants who reported use of antiplatelet agents other than ASA were included in the ASA users' group. Information on self-reported regular medical visits was also obtained; a regular medical visit was defined as at least 1 routine medical examination in the previous year.

Sociodemographic variables collected included age, sex, income and education. Annual income was stratified into 3 categories: low $(<\$ 50000)$, medium $(\$ 50000-\$ 150000)$ and high (>\$150 000). Similarly, 3 education categories were created: low (high school or less), medium (college) and high (university).

Blood pressure values were obtained by calculating the mean of 3 measurements taken with an automated device by means of an oscillometric method. Blood samples were collected for lipid profile measurement, used to compute the Framingham Risk Score. Obesity was defined as a body mass index of 30 or greater. Smoking status was classified into 4 categories: never smoked (lifetime smoking history $<100$ cigarettes), active smoker ( $\geq 1$ cigarette daily in previous $30 \mathrm{~d}$ ), occasional smoker ( $\geq 1$ cigarette in previous $30 \mathrm{~d}$ ) and past smoker (no cigarette in previous $30 \mathrm{~d}$ ).

\section{Statistical analysis}

Primary analyses were restricted to the subgroup of participants eligible for antiplatelet treatment for primary CVD prevention, as defined in the US Preventive Services Task Force recommendations. ${ }^{8}$ We determined factors associated with ASA use using logistic regression. We performed $\chi^{2}$ tests for categorical variables and trend tests for ordinal variables. We conducted logistic regression analyses to identify variables related to ASA use. Variables that were included in the model were age, sex, regular medical visits, education, income, smoking status, obesity, hypertension, diabetes and dyslipidemia. We assessed discrimination of the model by the C-statistic and calibration by the Hosmer-Lemeshow goodness-of-fit 
test. Statistical tests were 2 -sided, and a $p$ value $<0.05$ was considered to represent statistical significance. In the subgroup of participants with no history of CVD, we performed multiple imputation (chained equation imputation) for missing values of the following variables: Framingham Risk Score, education, smoking, obesity and annual income. We performed all analyses using SPSS software, version 24 (IBM Corporation).

\section{Ethics approval}

The study was approved by the Hôpital du Sacré-Cœur de Montréal Research Ethics Committee. All CARTaGENE participants signed a consent form, and the study adhered to the Declaration of Helsinki.

\section{Results}

The eligibility of CARTaGENE participants for ASA use for primary CVD prevention is shown in Figure 1. Of the 20004 participants, we excluded 1237 (6.2\%) with a past his-

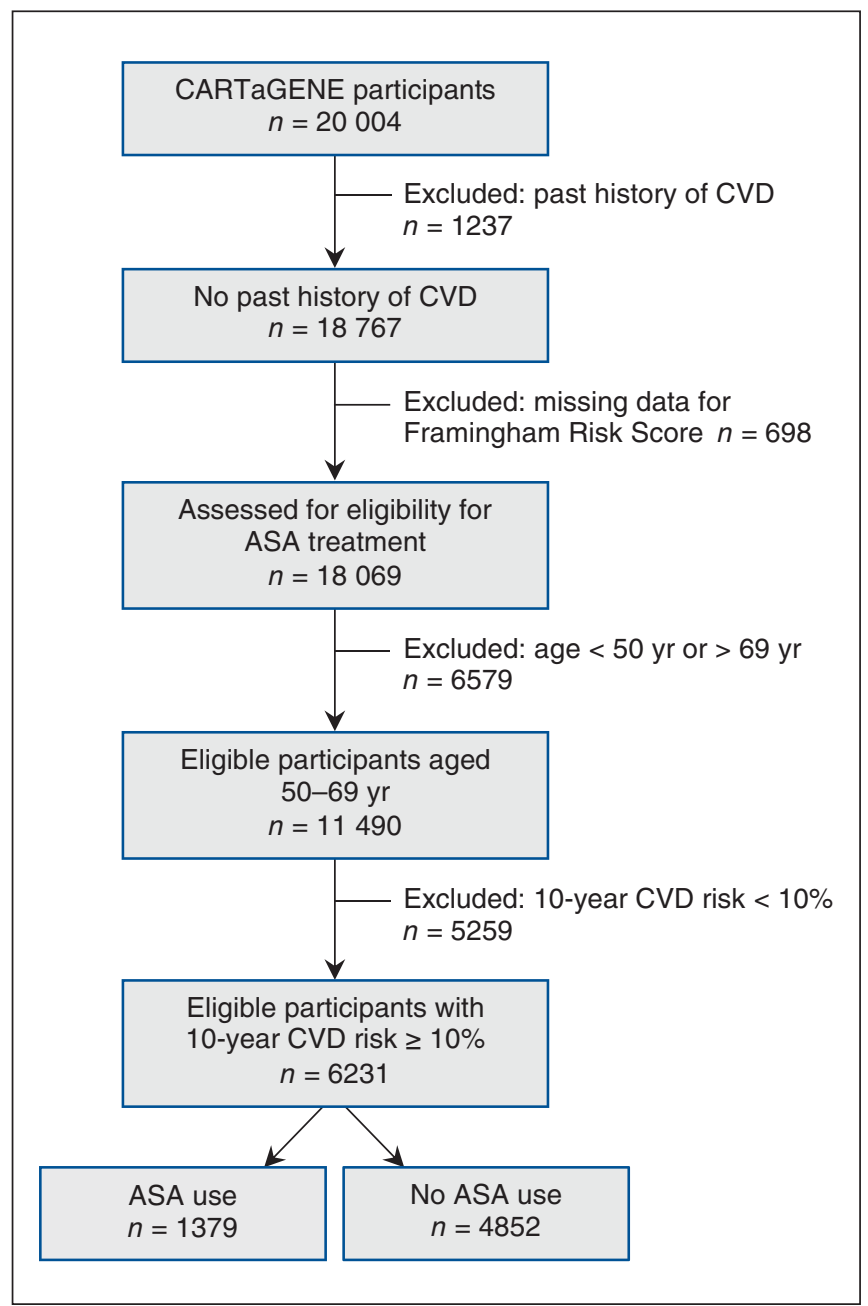

Figure 1: Flow diagram showing eligibility of CARTaGENE study ${ }^{13}$ participants for acetylsalicylic acid (ASA) use in primary prevention of cardiovascular disease (CVD) according to the US Preventive Services Task Force criteria. ${ }^{8}$ tory of CVD, of whom $504(40.7 \%)$ reported a history of myocardial infarction alone, $274(22.2 \%)$ stroke alone, 55 (4.4\%) both, and 404 (32.7\%) angina alone. Regular use of ASA was reported by 906 (73.2\%) of those with prior CVD. We excluded participants for whom the Framingham Risk Score could not be calculated owing to missing data $(n=698)$.

Applying the US Preventive Services Task Force eligibility criteria identified 6231 potential candidates for ASA use in primary CVD prevention (54.2\% of the 11490 participants aged 50-69 years with no past history of CVD) (Figure 1). The prevalence of antiplatelet therapy was low among eligible participants (1379 [22.1\%]). Of the 1379, $21(1.5 \%)$ reported using clopidogrel instead of ASA, and 9 reported using both ASA and clopidogrel. Of the 4852 participants not receiving any antiplatelet agent, $58(1.2 \%)$ were receiving an anticoagulant medication, which contraindicates the use of ASA for primary CVD prevention.

Key baseline characteristics of CARTaGENE participants eligible for preventive ASA use according to US Preventive Services Task Force recommendations are presented in Table 1. Eligible candidates had a mean age of 60.1 (standard deviation 5.7) years, and the majority (4489 [72.0\%]) were men. A total of 787 participants $(12.6 \%)$ reported a diagnosis of diabetes, $2512(40.3 \%)$ a diagnosis of hypertension, and $2375(38.1 \%)$ a diagnosis of dyslipidemia.

Table 2 presents factors associated with ASA use. There were significant associations between ASA use and all studied variables except for sex. Of the 787 eligible participants with diabetes, 478 (60.7\%) reported using ASA, and a majority of these participants were at high risk for CVD (Framingham Risk Score $\geq 20 \%$ in 325 [68.0\%], obesity in 272 [56.9\%], hypertension in 319 [66.7\%] and dyslipidemia in 337 [70.5\%]).

Multivariate analyses performed to identify factors associated with ASA use showed that ASA use was positively associated with older age, male sex, regular medical visits, lower education level, obesity, hypertension, diabetes and dyslipidemia (Table 3). Income and smoking status were not found to be significantly associated with ASA use. When these analyses were repeated after multiple imputation for missing values, there were no meaningful differences identified in the risk factors or the odd ratios.

Our results indicate that 885261 people would potentially have started ASA treatment if the US Preventive Services Task Force recommendations had been implemented in Quebec in 2018 (Figure 2). ${ }^{15}$

\section{Interpretation}

Using US Preventive Services Task Force criteria ${ }^{8}$ in a large representative population-based sample from Quebec, we found that a majority of people from the targeted age group with no prior CVD history were eligible for preventive ASA use but that, among these participants, the prevalence of ASA use was low (22\%). Implementation of these recommendations in Quebec would thus require initiating ASA for a substantial number of people. We identified factors associated with ASA use, some of which (high 
Table 1: Baseline characteristics of CARTaGENE study ${ }^{13}$ participants eligible for acetylsalicylic acid therapy for primary prevention of cardiovascular disease

\begin{tabular}{|c|c|}
\hline Characteristic & $\begin{array}{l}\text { No. (\%) of participants } \\
\qquad n=6231\end{array}$ \\
\hline \multicolumn{2}{|l|}{ Age group, yr } \\
\hline $50-59$ & $2989(48.0)$ \\
\hline $60-69$ & $3242(52.0)$ \\
\hline Male sex & $4489(72.0)$ \\
\hline \multicolumn{2}{|l|}{ Annual income* } \\
\hline Low & 2359 (37.9) \\
\hline Medium & $2946(47.3)$ \\
\hline High & $501(8.0)$ \\
\hline Missing & $425(6.8)$ \\
\hline \multicolumn{2}{|l|}{ Education† } \\
\hline Low & $1882(30.2)$ \\
\hline Medium & $1871(30.0)$ \\
\hline High & 2425 (38.9) \\
\hline Missing & $53(0.8)$ \\
\hline Regular medical visits & 4447 (71.4) \\
\hline \multicolumn{2}{|l|}{ Obesity } \\
\hline Yes & 1997 (32.0) \\
\hline No & $4122(66.2)$ \\
\hline Missing & $112(1.8)$ \\
\hline Hypertension $\ddagger$ & $2512(40.3)$ \\
\hline Diabetesł & 787 (12.6) \\
\hline Dyslipidemiał & $2375(38.1)$ \\
\hline \multicolumn{2}{|l|}{ Smoking category } \\
\hline Active & $1165(18.7)$ \\
\hline Stopped & $2739(44.0)$ \\
\hline Never & $2056(33.0)$ \\
\hline Occasional & $220(3.5)$ \\
\hline Missing & $51(0.8)$ \\
\hline $\begin{array}{l}\text { *Low }=\text { less than } \$ 50000, \mathrm{~m} \\
\$ 150000 . \\
\dagger \text { Low }=\text { high school or less, } \\
\text { †Self-reported. }\end{array}$ & $\begin{array}{l}000 \$ \text {, high = more than } \\
=\text { university. }\end{array}$ \\
\hline
\end{tabular}

blood pressure, diabetes and dyslipidemia) increased the odds of prophylactic ASA use more than twofold.

Other estimates of preventive ASA use in various cohorts have previously been published, with rates as low as $3 \%$ in a Swedish cohort of 5725 participants ${ }^{16}$ and as high as $47 \%$ in a US Web-based survey. ${ }^{12}$ In a study by Fiscella and colleagues 9 using National Health and Nutrition Examination Survey data, $87 \%$ of men aged $45-79$ years and $16 \%$ of women aged 55-79 years were eligible for antiplatelet therapy according to the 2009 US Preventive Services Task Force recommendations, ${ }^{17}$ but only $32 \%$ of eligible men and $47 \%$ of eligible women reported current ASA use. The prevalence of ischemic vascular disease in the CARTaGENE sample (6.2\%) is equiv-
Table 2: Acetylsalicylic acid use according to demographic and clinical characteristics

\begin{tabular}{|c|c|c|}
\hline Characteristic & $\begin{array}{c}\text { No. }(\%) \text { who used } \\
\text { ASA }^{*}\end{array}$ & $p$ value $\dagger$ \\
\hline Age group, yr & & $<0.001$ \\
\hline $50-59$ & $462(15.5)$ & \\
\hline $60-69$ & $917(28.3)$ & \\
\hline Sex & & 0.4 \\
\hline Male & $1006(22.4)$ & \\
\hline Female & $373(21.4)$ & \\
\hline Income & & 0.02 \\
\hline Low & $552(23.4)$ & \\
\hline Medium & $629(21.4)$ & \\
\hline High & $98(19.6)$ & \\
\hline Education & & 0.002 \\
\hline Low & $457(24.3)$ & \\
\hline Medium & $414(22.1)$ & \\
\hline High & $494(20.4)$ & \\
\hline Regular medical visits & & $<0.001$ \\
\hline Yes & $1157(26.0)$ & \\
\hline No & $222(12.4)$ & \\
\hline Obesity & & $<0.001$ \\
\hline Yes & $610(30.5)$ & \\
\hline No & $742(18.0)$ & \\
\hline Hypertension & & $<0.001$ \\
\hline Yes & $869(34.6)$ & \\
\hline No & $510(13.7)$ & \\
\hline Diabetes & & $<0.001$ \\
\hline Yes & $478(60.7)$ & \\
\hline No & $901(16.6)$ & \\
\hline \multicolumn{3}{|l|}{ Dyslipidemia } \\
\hline Yes & $849(35.7)$ & \\
\hline No & $530(13.7)$ & \\
\hline Smoking category & & $<0.001$ \\
\hline Active & $198(17.0)$ & \\
\hline Stopped & $676(24.7)$ & \\
\hline Never & $446(21.7)$ & \\
\hline Occasional & $47(21.4)$ & \\
\hline $\begin{array}{l}\text { Note: } A S A=\text { acetylsalicylic } \\
{ }^{*} \text { ASA or other platelet aggre } \\
\text { †For difference in the propo }\end{array}$ & $\begin{array}{l}\text { bitor(s). } \\
\text { A users between subs }\end{array}$ & \\
\hline
\end{tabular}

alent to that reported in a Canadian survey ${ }^{18}$ but somewhat lower than that reported in surveys from the US with similar designs. ${ }^{10-12}$

Multiple factors may explain the low rate of ASA use in the present survey. First, recommendations on ASA use for primary CVD prevention were conflicting at the time of the study (2010) and still are today (Table 4). For instance, in 
Table 3: Factors associated with acetylsalicylic acid use for primary prevention of cardiovascular disease among eligible participants on multivariate analysis

\begin{tabular}{|lc|}
\hline Variable $^{*}$ & OR $(95 \% \mathrm{Cl})$ \\
\hline Age (per year) & $1.08(1.06-1.09)$ \\
\hline Male sex & $1.51(1.28-1.79)$ \\
\hline Regular medical visits & $1.56(1.30-1.87)$ \\
\hline Education & $1.20(1.01-1.42)$ \\
\hline \multicolumn{1}{|c|}{ Low } & $1.21(1.02-1.44)$ \\
\hline Medium & Reference \\
\hline \multicolumn{1}{|c|}{ High } & $1.38(1.19-1.60)$ \\
\hline Obesity & $2.41(2.08-2.80)$ \\
\hline Hypertension & $5.48(4.56-6.60)$ \\
\hline Diabetes & $2.41(2.09-2.78)$ \\
\hline Dyslipidemia & \\
\hline $\begin{array}{l}\text { Note: Cl }=\text { confidence interval, OR }=\text { odds ratio. } \\
\text { *Income and smoking category were also included in the multivariate model but } \\
\text { are not presented in the table because no significant association was found with } \\
\text { acetylsalicylic acid use. }\end{array}$ \\
\hline
\end{tabular}

contrast to the 2016 US Preventive Services Task Force recommendations, ${ }^{8}$ the US Food and Drug Administration does not support ASA use as a primary prevention strategy to reduce the burden of CVD. ${ }^{21}$ In its 2011 guideline, the Canadian Cardiovascular Society did not recommend the routine use of ASA but suggested that it could be considered for those at high vascular risk. ${ }^{25}$

What explains such discrepancies between guidelines? Large meta-analyses of primary prevention trials ${ }^{3,26-30}$ suggest that ASA lowers the risk of major CVD events (hazard ratio $0.89,95 \%$ confidence interval $0.84-0.94),{ }^{29}$ which is driven mainly by a reduction in the risk of nonfatal myocardial infarction. Studies have also shown potential long-term benefits of ASA use on colorectal cancer incidence and mortality when taken for at least 10 years. ${ }^{31} \mathrm{~A}$ recent meta-analysis, however, did not show any reduced incidence of colorectal cancer. ${ }^{29}$ The net impact of these results in terms of absolute benefits depends on the baseline risk of ischemic vascular events, and these potential benefits must be weighed against the inherent risks associated with daily ASA use, mainly risk of bleeding (hazard ratio 1.43, 95\% confidence interval 1.30-1.56). ${ }^{29}$ More recent data from the ASCEND ${ }^{5}$ and

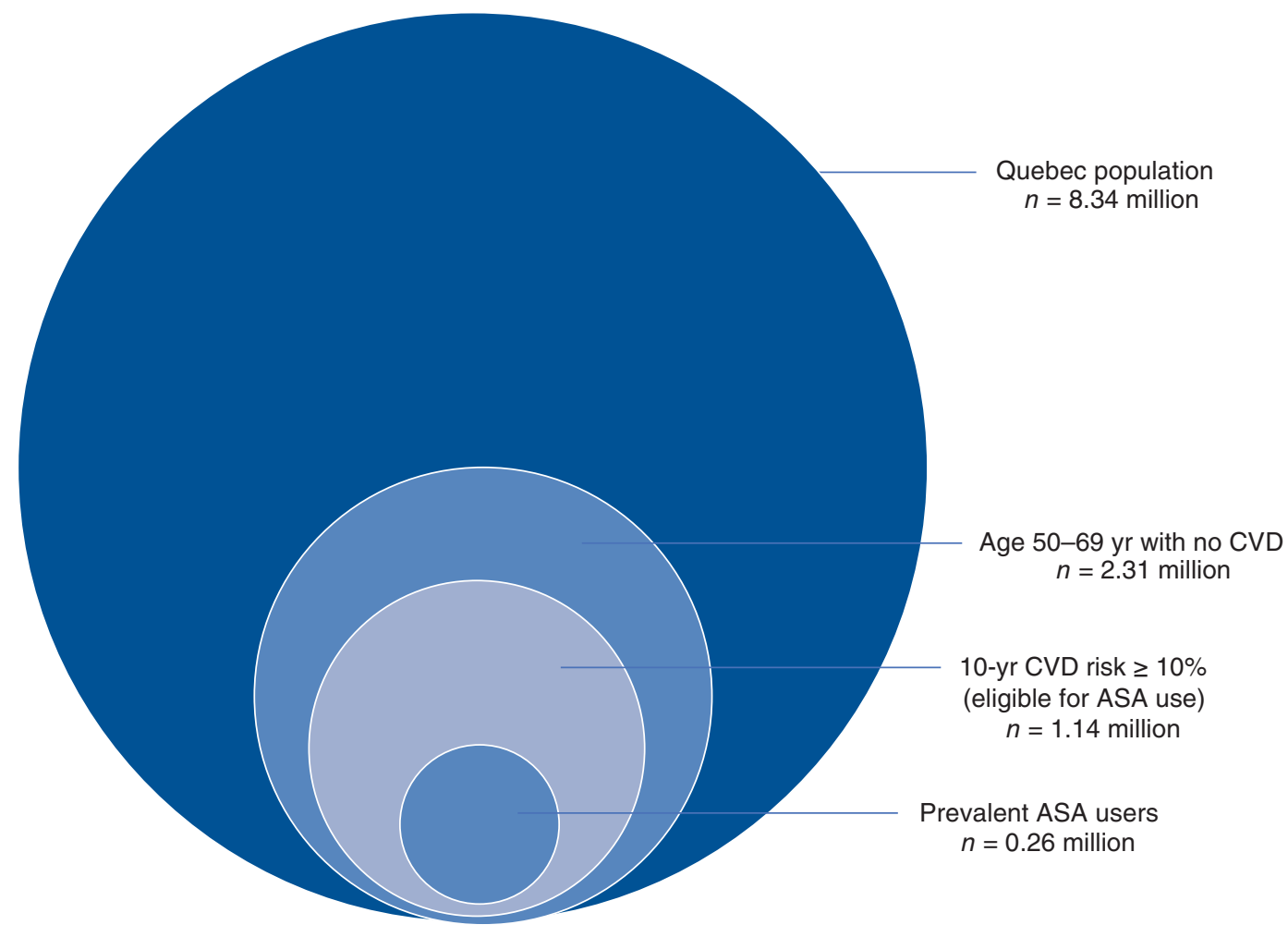

Figure 2: Estimate of the Quebec population eligible for acetylsalicylic acid (ASA) use for primary prevention of cardiovascular disease (CVD) according to the 2016 US Preventive Services Task Force recommendations ${ }^{8}$ and prevalent users. Implementation of the recommendations would necessitate initiation of ASA treatment in an additional 0.88 million people (1.14 million eligible people - 0.26 million prevalent ASA users). 


\begin{tabular}{|c|c|}
\hline Organization, year & Recommendation \\
\hline $\begin{array}{l}\text { American College of Cardiology/ } \\
\text { American Heart Association, } 2019^{7}\end{array}$ & Consider for adults aged $40-70 \mathrm{yr}$ at high risk \\
\hline Hypertension Canada, $2018^{19}$ & Consider for hypertensive adults aged $\geq 50 \mathrm{yr}$ \\
\hline $\begin{array}{l}\text { Canadian Diabetes Association, } \\
2018^{23}\end{array}$ & $\begin{array}{l}\text { Do not use routinely, but may be used in presence } \\
\text { of additional cardiovascular risk factors other than } \\
\text { diabetes }\end{array}$ \\
\hline $\begin{array}{l}\text { US Preventive Services Task Force, } \\
2016^{8}\end{array}$ & $\begin{array}{l}\text { Initiate for adults aged } 50-59 \text { yr with a } 10 \text {-year CVD } \\
\text { risk } \geq 10 \% \text {; individualize decision for adults aged } \\
60-69 \text { yr with a } 10 \text {-year CVD risk } \geq 10 \%\end{array}$ \\
\hline $\begin{array}{l}\text { Sixth Joint Task Force of the } \\
\text { European Society of Cardiology and } \\
\text { Other Societies, } 2016^{20}\end{array}$ & Do not initiate \\
\hline $\begin{array}{l}\text { US Food and Drug Administration, } \\
2014^{21}\end{array}$ & Do not initiate \\
\hline $\begin{array}{l}\text { American Heart Association/ } \\
\text { American Stroke Association, } 2014^{22}\end{array}$ & ASA use is reasonable if 10 -year CVD risk is $\geq 10 \%$ \\
\hline $\begin{array}{l}\text { American College of Chest } \\
\text { Physicians, } 2012^{24}\end{array}$ & Suggested for adults aged $\geq 50 \mathrm{yr}$ \\
\hline $\begin{array}{l}\text { Canadian Cardiovascular Society, } \\
2011^{25}\end{array}$ & Consider if high vascular risk \\
\hline $\begin{array}{l}\text { US Preventive Services Task Force, } \\
2009^{17}\end{array}$ & $\begin{array}{l}\text { Encourage men aged } 45-79 \text { yr and women aged } \\
55-79 \text { yr to use ASA when potential CVD benefit } \\
\text { outweighs potential harm (based on 10-year CVD } \\
\text { risk) }\end{array}$ \\
\hline
\end{tabular}

ARRIVE $^{6}$ trials are consistent with these previous trials. The relative benefits of daily ASA use compared to risks appear limited in recent trials, ${ }^{5,6}$ leading to a lower class of recommendation in the latest American College of Cardiology/ American Heart Association guideline. ${ }^{7}$

Weighing benefits and harms of ASA use in primary prevention of CVD for a given patient undoubtedly remains a challenge. One major finding of recent trials is the overestimation of CVD risk from risk calculators. For instance, in the ARRIVE trial, ${ }^{6}$ which aimed to study prophylactic ASA use in a population at moderate risk for CVD, total events were lower than predicted by risk scores. Further studies will need to strive to identify more accurate ways of defining a subpopulation of patients with increased baseline CVD risk who would likely benefit from ASA use.

\section{Limitations}

There are some limitations to our study. The methodology for recruitment of CARTaGENE participants may have introduced a selection bias; however, overall, the final cohort was shown to be representative of the general population of the province of Quebec. Moreover, many variables were selfreported, as is the case in most similar studies, which potentially limits the exactitude of collected data. For instance, the presence of CVD was self-reported, which may have led to some misclassification. However, unlike other researchers, the
CARTaGENE investigators included extensive data from biologic sampling and physiologic measurements, so that CVD risk scores could be calculated for most respondents.

The detailed questionnaire completed by participants could not capture cases in which a deliberate decision was made by a physician not to prescribe ASA after evaluation of benefits and harms of such a treatment. In some instances, this decision might also have originated from the participant's preferences. Similarly, for respondents who reported regular antiplatelet use, the questionnaire could not ascertain the main purpose of such use, which might not always have been primary CVD prevention. Furthermore, our evaluation of potential contraindications to antiplatelet therapy was incomplete and our assessment of bleeding risk limited. However, we explored data on anticoagulation, which showed that only $1.2 \%$ of people at high risk not receiving preventive ASA were taking an anticoagulant agent and thus had a contraindication to ASA use for primary CVD prevention.

When enrolment in CARTaGENE began, different guidelines were in place. For instance, the 2009 US Preventive Services Task Force recommendations ${ }^{17}$ differed from the updated 2016 recommendations ${ }^{8}$ in that preventive ASA use was recommended based on different proposed cut-offs of 10 -year CVD risk estimates based on age and sex. Finally, there were missing data. However, the proportion of missing values for most variables was very small, and when the 
analyses were repeated after multiple imputation for missing values, there were no meaningful differences identified in the risk factors or the odd ratios.

\section{Conclusion}

We showed that the use of ASA for primary CVD prevention was low in a large Canadian population-based survey. Strict implementation of 2016 US Preventive Services Task Force recommendations would require initiating ASA treatment in a substantial proportion of people, with undetermined potential benefits.

\section{References}

1. Cardiovascular diseases (CVDs). Geneva: World Health Organization; 2017. Available: www.who.int/news-room/fact-sheets/detail/cardiovascular-diseases -(cvds) (accessed 2019 July 5).

2. Table 13-10-0394-01: Leading causes of death, total population, by age group. Ottawa: Statistics Canada; modified 2019 Dec. 2. Available: www150.statcan. gc.ca/t1/tbl1/en/tv.action?pid=1310039401 (accessed 2019 July 5),

3. Antithrombotic Trialists' (ATT) Collaboration; Baigent C, Blackwell L, Collins R, et al. Aspirin in the primary and secondary prevention of vascular disease: collaborative meta-analysis of individual participant data from randomised trials. Lancet 2009;373:1849-60.

4. Antithrombotic Trialists Collaboration. Collaborative meta-analysis of randomised trials of antiplatelet therapy for prevention of death, myocardial infarction, and stroke in high risk patients [published erratum in BMF 2002; 324:141]. BM7 2002;324:71-86.

5. ASCEND Study Collaborative Group; Bowman L, Mafham M, Wallendszus $\mathrm{K}$, et al. Effects of aspirin for primary prevention in persons with diabetes mellitus. N Engl 7 Med 2018;379:1529-39.

6. Gaziano JM, Brotons C, Coppolecchia R, et al.; ARRIVE Executive Committee. Use of aspirin to reduce risk of initial vascular events in patients at moderate risk of cardiovascular disease (ARRIVE): a randomised, double-blind, placebo-controlled trial. Lancet 2018;392:1036-46.

7. Arnett DK, Blumenthal RS, Albert MA, et al. 2019 ACC/AHA guideline on the primary prevention of cardiovascular disease: a report of the American College of Cardiology/American Heart Association Task Force on clinical practice guidelines. Circulation 2019;140:e596-646.

8. Bibbins-Domingo K; U.S. Preventive Services Task Force. Aspirin use for the primary prevention of cardiovascular disease and colorectal cancer: U.S. Preventive Services Task Force recommendation statement. Ann Intern Med 2016; 164:836-45.

9. Fiscella K, Winters PC, Mendoza M, et al. Do clinicians recommend aspirin to patients for primary prevention of cardiovascular disease? 7 Gen Intern Med 2015;30:155-60

10. Pignone $M$, Anderson GK, Binns K, et al. Aspirin use among adults aged 40 and older in the United States: results of a national survey. Am 7 Prev Med 2007;32:403-7.

11. Gu Q, Dillon CF, Eberhardt MS, et al. Preventive aspirin and other antiplatelet medication use among U.S. adults aged $\geq 40$ years: data from the National Health and Nutrition Examination Survey, 2011-2012. Public Health Rep 2015; 130:643-54.

12. Williams CD, Chan AT, Elman MR, et al. Aspirin use among adults in the U.S.: results of a national survey. Am 7 Prev Med 2015;48:501-8.

13. Awadalla P, Boileau C, Payette Y, et al.; CARTaGENE Project. Cohort profile of the CARTaGENE study: Quebec's population-based biobank for public health and personalized genomics. Int 7 Epidemiol 2013;42: 1285-99.

14. D'Agostino RB Sr, Vasan RS, Pencina MJ, et al. General cardiovascular risk profile for use in primary care: the Framingham Heart Study. Circulation 2008; 117:743-53.

15. Le bilan démographique du Québec: édition 2018. Québec: Institut de la statistique du Québec; 2018. Available: www.stat.gouv.qc.ca/statistiques/population -demographie/bilan2018.pdf (accessed 2019 July 5).

16. Rodondi N, Cornuz J, Marques-Vidal P, et al. Aspirin use for the primary prevention of coronary heart disease: a population-based study in Switzerland. Prev Med 2008:46:137-44

17. US Preventive Services Task Force. Aspirin for the prevention of cardiovascular disease: U.S. Preventive Services Task Force recommendation statement. Ann Intern Med 2009;150:396-404.

18. CCDI Steering Committee. The 2017 Canadian Chronic Disease Indicators [published erratum in Health Promot Chronic Dis Prev Can 2017;37:262]. Health Promot Chronic Dis Prev Can 2017;37:248-51.
19. Nerenberg KA, Zarnke KB, Leung AA, et al.; Hypertension Canada. Hypertension Canada's 2018 guidelines for diagnosis, risk assessment, prevention, and treatment of hypertension in adults and children. Can 7 Cardiol 2018;34:506-25.

20. Piepoli MF, Hoes AW, Agewall S, et al.; ESC Scientific Document Group. 2016 European Guidelines on cardiovascular disease prevention in clinical practice: the Sixth Joint Task Force of the European Society of Cardiology and Other Societies on Cardiovascular Disease Prevention in Clinical Practice (constituted by representatives of 10 societies and by invited experts) developed with the special contribution of the European Association for Cardiovascular Prevention \& Rehabilitation (EACPR). Eur Heart 7 2016;37:2315-81.

21. Use of aspirin for primary prevention of heart attack and stroke. Silver Spring (MD): US Food and Drug Administration; 2014. Available: www.fda.gov/ drugs/resourcesforyou/consumers/ucm390574.htm (accessed 2019 July 5).

22. Meschia JF, Bushnell C, Boden-Albala B, et al.; American Heart Association Stroke Council; Council on Cardiovascular and Stroke Nursing; Council on Clinical Cardiology; Council on Functional Genomics and Translational Biology; Council on Hypertension. Guidelines for the primary prevention of stroke: a statement for healthcare professionals from the American Heart Association/American Stroke Association. Stroke 2014;45:3754-832.

23. Diabetes Canada Clinical Practice Guidelines Expert Committee; Stone JA Houlden RL, Lin P, et al. Cardiovascular protection in people with diabetes [published erratum in Can 7 Diabetes 2019;43:154]. Can 7 Diabetes 2018;42 (Suppl 1):S162-9.

24. Vandvik PO, Lincoff AM, Gore JM, et al. Primary and secondary prevention of cardiovascular disease: Antithrombotic Therapy and Prevention of Thrombosis, 9th ed: American College of Chest Physicians Evidence-Based Clinical Practice Guidelines. Chest 2012;141(Suppl):e637S-68S.

25. Bell AD, Roussin A, Cartier R, et al.; Canadian Cardiovascular Society. The use of antiplatelet therapy in the outpatient setting: Canadian Cardiovascular Society guidelines. Can 7 Cardiol 2011;27(Suppl A):S1-59.

26. Bartolucci AA, Tendera M, Howard G. Meta-analysis of multiple primary prevention trials of cardiovascular events using aspirin. Am f Cardiol 2011;107:1796-801.

27. Seshasai SR, Wijesuriya S, Sivakumaran R, et al. Effect of aspirin on vascular and nonvascular outcomes: meta-analysis of randomized controlled trials. Arch Intern Med 2012;172:209-16.

28. Guirguis-Blake JM, Evans CV, Senger CA, et al. Aspirin for the primary prevention of cardiovascular events: a systematic evidence review for the U.S. Preventive Services Task Force. Ann Intern Med 2016;164:804-13.

29. Zheng SL, Roddick AJ. Association of aspirin use for primary prevention with cardiovascular events and bleeding events: a systematic review and metaanalysis. FAMA 2019;321:277-87.

30. Mahmoud AN, Gad MM, Elgendy AY, et al. Efficacy and safety of aspirin for primary prevention of cardiovascular events: a meta-analysis and trial sequential analysis of randomized controlled trials. Eur Heart 7 2019;40:607-17.

31. Chubak J, Kamineni A, Buist DSM, et al. Aspirin use for the prevention of colorectal cancer: an updated systematic evidence review for the U.S. Preventive Services Task Force. U.S. Preventive Services Task Force Evidence Syntheses, Formerly Systematic Evidence Reviews. Rockville (MD): Agency for Healthcare Research and Quality (US); 2015. Report no 15-05228-EF-1.

Affiliations: Faculty of Medicine (Khalili, Lepeytre, Goupil, Troyanov, Bouchard, Madore), Université de Montréal; Centre de recherche de l'Hôpital du Sacré-Cœur de Montréal (Khalili, Lepeytre, Goupil, Troyanov, Bouchard, Madore), Montréal, Que.; Département de médecine sociale et preventive (Guertin), Faculty of Medicine, Université Laval; Centre de recherche du Centre hospitalier universitaire de Québec (Guertin), Québec, Que.

Contributors: François Madore supervised the project. Myriam Khalili, Fanny Lepeytre and François Madore conceived the study. Myriam Khalili, Fanny Lepeytre, Jason Robert Guertin and François Madore designed the study and analyzed the data. Myriam Khalili, François Madore and Jason Robert Guertin drafted the manuscript. All of the authors contributed to interpretation of data, revised the manuscript critically for important intellectual content, approved the final version to be published and agreed to be accountable for all aspects of the work.

Funding: This work was supported by personal research funds from the authors. CARTaGENE was supported by Genome Canada, Génome Québec and the Quebec Ministry of Health. Rémi Goupil and Jason Robert Guertin hold research scholarships from the Fonds de recherche du Québec - Santé.

Supplemental information: For reviewer comments and the original submission of this manuscript, please see www.cmajopen.ca/content/8/1/ E41/suppl/DC1. 